\title{
Representações sociais de puérperas sobre a adesão ao pré-natal na fronteira franco-brasileira
}

\author{
Lise Maria Carvalho Mendes', Graciano Almeida Sudré1, Jordânia \\ Vieira Oliveira ${ }^{2}$, Nayara Gonçalves Barbosa ${ }^{1}$, Juliana Cristina dos \\ Santos Monteiro' e Flavia Azevedo Gomes-Sponholz ${ }^{1}$
}

${ }^{1}$ Programa de Pós-Graduação em Enfermagem em Saúde Pública. Universidade de São Paulo, Brasil | lisedemendes@usp.br; gracianosudre@usp.br; nagbarbosa@gmail.com; jumonte@eerp.usp.br; flagomes@eerp.usp.br | http://orcid.org/0000-0001-9325-8382; http://orcid.org/0000-0003-2837-8526; http://orcid.org/0000-0003-3646-4133; http://orcid.org/0000-0001-6470-673X; http://orcid.org/0000-0003-1540-0659

2 Departamento de Ciências Biológicas e Saúde. Universidade Federal do Amapá, Brasil | jordaniavieiragje@gmail.com | http://orcid.org/0000-0001-5308-1009

Resumo: Introdução: No Brasil, os indicadores da assistência pré-natal demonstram uma persistência da inadequação dos serviços de saúde. Esta inadequação é observada principalmente na região Amazônica, mediante contexto de desigualdades sociais e regionais. Desta forma, este estudo pretende compreender as representações sociais sobre a busca pela assistência pré-natal por puérperas que aderiram ao pré-natal tardiamente na região supracitada. Metodologia: estudo qualitativo, realizado com 13 puérperas, em Alojamento Conjunto. As entrevistas foram semi-diretivas, gravadas e transcritas na íntegra, organizadas a partir da Análise Temática. Resultados: Verificou-se as categorias: Percepção socialmente construídas da assistência pré-natal e Obstáculos que interferem na não-adesão ao pré-natal. Conclusões: A prevenção, o acolhimento e a aprendizagem são as principais percepções que motivam à realização do pré-natal. As representações dos obstáculos à adesão inseremse nas precárias relações trabalhistas, violência de gênero, variabilidade linguística e culturais; a ausência das redes de apoio e desorganização dos serviços.

Palavras-chave: Serviços de Saúde Materna; Saúde na Fronteira; Saúde Materna.

Social Representations of Puerperal Women about the Search for Prenatal Care on the French-Brazilian Border

\begin{abstract}
Introduction: In Brazil, prenatal care indicators demonstrate the persistence of inadequate health services. This inadequacy is observed mainly in the Amazon region, in a context of social and regional inequalities. Aims: to understand the social representations about the search for prenatal care for puerperal women who joined the prenatal care late. Methods: qualitative study, carried out with 13 puerperal women, in rooming-in. The centralized ones were semi-guidelines, recorded and transcribed in full, organized based on Thematic Analysis. Results: The following categories were verified: Socially constructed perception of prenatal care and Obstacles that interfere with non-adherence to prenatal care. Conclusions: Prevention, reception and learning are the main perceptions that motivate prenatal care. Representations of the rights to adherence are inserted in precarious work relationships, gender violence, linguistic and cultural variability; the absence of support networks and disorganization of services.
\end{abstract}

Keywords: Maternal Health Services; Border Health; Maternal Health.

\section{Introdução}

A assistencia pré-natal iniciada precocemente é associada a menores chances de complicações no período gravídico-puerperal. No entanto, observa-se que em diversas localidades ainda persistem barreiras de acesso a este tipo de assistência. Em locais que inexistem sistema de saúde público a principal barreira de acesso é a ausência de recursos financeiros (Ansu-Mensah, Danquah, Bawontuo, Ansu-Mensah, \& Kuupiel, 2020).

No contexto brasileiro, os serviços pré-natais estão presentes no escopo das ações realizadas pelo Sistema Único de Saúde (SUS). 
No entanto, persistem inadequações da assistência associadas às desigualdades sociais e regionais (Guimarães, Parente, Guimarães, \& Garnelo, 2018). Este cenário é visualizado principalmente na região Amazônica, entre os povos da floresta, em que se observa adesão tardia, número de consultas inferior a seis e altos índices de morbimortalidade materna e perinatal (Tomasi et al., 2017).

Mediante as iniquidades e disparidades regionais inseridas no contexto brasileiro, este trabalho busca desvelar a representação de puérperas sobre $o$ atendimento ofertado pelo SUS na região supracitada, a partir do questionamento: Quais as representações sociais das mulheres que aderiram ao pré-natal tardiamente sobre o pré-natal? A TRS aplicada às pesquisas de enfermagem busca compreender as representações estabelecidas sobre o cuidado, além de conhecer os significados a ele atribuídos. Para tanto, observa os contextos que sustentam estes significados, o que nos aproxima da compreensão de comportamentos, das atitudes e das escolhas das pessoas referentes aos cuidados de saúde, auxiliando a conduzir o cuidado num plano terapêutico que mais se aproxima do outro a quem o cuidado se destina (Silva, Mendes, Monteiro, \& Silva, 2019).

Desta forma, este estudo objetivou compreender as representações sociais sobre a busca pela assistência pré-natal por puérperas que aderiram ao pré-natal tardiamente. Diante dos fatores subjetivos contidos na relação entre o indivíduo que recebe o cuidado e 0 sistema que o produz, este estudo visa subsidiar reflexões importantes e estimuladoras para o redirecionamento dos programas e dos serviços de saúde.

\section{Metodologia}

Estudo qualitativo, descritivo, em que utilizou-se a Teoria das Representações Sociais (TRS) como referencial teórico. Entende-se por TRS a forma como os grupos sociais interpretam a realidade que vivenciam, formulam seus conhecimentos e orientam as suas condutas e práticas, com base nos valores que atribuem às suas vivências (Moscovici, 2003).

Em relação ao local de estudo, essa pesquisa foi desenvolvida no alojamento conjunto do Hospital Estadual de Oiapoque (HEO), instituição pública de média complexidade situada no município de Oiapoque-Amapá, situado na fronteira entre Brasil e Guiana Francesa. Este município possui 24 mil habitantes, dos quais cerca de 8 mil são indígenas. O município localiza-se a $590 \mathrm{~km}$ da capital Macapá e seu acesso se dá por uma rodovia não completamente pavimentada, o que, durante o período de inverno amazônico, dificulta a locomoção de seus habitantes à capital, o que torna o HEO a única instituição de saúde a que podem recorrer em caso de necessidade de assistência hospitalar.

As participantes foram mulheres brasileiras, no período de puerpério imediato, residentes nesta faixa de fronteira, que se encontravam no ambiente hospitalar e que aderiram ao pré-natal tardiamente no último ciclo gravídico. Considerou-se adesão tardia ao pré-natal as consultas iniciadas após $12^{a}$ semana de gestação, conforme orientação do Ministério da Saúde. Foram excluídas puérperas com alterações psíquicas, complicações obstétricas e neonatais durante o trabalho de parto, parto e pós-parto. Foram convidadas 15 puérperas, nas quais 13 foram entrevistadas. A seleção das puérperas convidadas foi pautada pela ordem de admissão na sala de pós-parto. Entre os motivos de recusa evidenciou-se a presença de melancolia, angústia e impotência pela não progressão do trabalho de parto ativo. Estabeleceu-se o critério de finalização das entrevistas a saturação teórica dos conteúdos, ou seja, quando o eixo discursivo central assumiu padrões de repetição.

A coleta de dados ocorreu em novembro de 2017, mediante a técnica de entrevista semidiretiva, em um processo de esclarecimento e aprofundamento das ideias construídas no encontro. As entrevistas foram realizadas por um pesquisador enfermeiro, com expertise em pesquisa qualitativa. A mesma ocorreu em local privativo guiadas pelos temas gerais: percepção sobre o pré-natal, consultas de pré-natal, busca tardia das consultas de prénatal. 
Foram audiogravadas, validadas e transcritas na íntegra, com duração média de 30 minutos. O material foi submetido à técnica de análise temática do conteúdo, descritas a seguir.

Esta etapa foi realizada por dois pesquisadores, de formações distintas, do campo da saúde, com domínio de análise de entrevistas qualitativas. A pré-análise constituiu-se por um primeiro contato através da leitura flutuante do material, seguido por uma leitura reflexiva para organização das unidades de registro e contexto, permitindo orientação e direcionamento das impressões. Na etapa de exploração do material, os pesquisadores realizaram a distribuição e o esquema de classificação. Foi realizada a organização de tendências do fenômeno investigado e a busca por pontos de convergências para categorizar as decodificações. No tratamento e interpretação dos resultados obtidos procedeu-se a codificação do conteúdo subjacente ao que estava sendo manifesto, articulando os objetivos do estudo com as tendências e conteúdo teórico abordado.

A reflexão e análise das categorias foram alicerçadas na fala dos agentes. Destaca-se que para a compreensão dos núcleos de sentido não basta uma análise da narrativa em si. Se faz necessário que haja uma aproximação das condições sociais e histórias de produção e circulação das concepções compartilhadas pelo grupo que thes deram origem. Neste processo, a compreensão do contexto em que a pessoa é levada a reagir a um estímulo possibilita elementos para o entendimento dos processos de formulação de suas representações. Para identificação das representações socais se faz necessário recorrer a dois conceitos que auxiliam o processo de compreensão das representações: a objetivação e a ancoragem. A objetivação é a fase figurativa, resultado da capacidade que o pensamento e a linguagem possuem em materializar o abstrato em um novo conceito a partir dos registros individuais existentes. É o percurso pelo qual as representações adquirem materialidade. Já a ancoragem trata-se da transformação de algo estranho e perturbador, que nos intriga, em nosso sistema particular de categorias e o compara com paradigmas de uma categoria que nós acreditamos ser apropriada (Moscovicci, 2003).

Este estudo respeitou as exigências nacionais e internacionais regulamentadoras de pesquisas envolvendo seres humanos, de acordo com a Resolução no 466/2012, por meio da assinatura do termo de consentimento livre e esclarecido. Esse estudo foi aprovado sob o parecer 2.155.712 do Comitê de Ética em Pesquisa. Para manter o sigilo dos participantes, as puérperas foram identificadas pela letra $E$, de entrevistadas, procedida com o número sequencial em que foram realizadas as entrevistas.

Foram identificadas as seguintes categorias analíticas, formuladas a partir das representações sociais apreendidas dos discursos, são elas: percepção socialmente construídas da assistência pré-natal, em que surgiram as subcategorias prevenção, aprendizagem e acolhimento; e obstáculos que interferem na não-adesão ao pré-natal, que se subdividiu em outros três eixos analíticos ausência de rede de apoio, aspectos socioculturais e desorganização dos serviços de saúde.

\section{Resultados e Discussão}

A disposição dos dados foi norteada pelo mapeamento de ideias centrais e das representações sociais, em que foram verificadas categorias e subcategorias, de acordo com a Tabela 1. 
Tabela 1. Mapa das Associações de ideias sobre pré-natal presente nos discursos das puérperas. Oiapoque-AP, Brasil, 2017.

\begin{tabular}{lll}
\hline Categorias & Subcategorias & Falas \\
\hline
\end{tabular}

1. Percepção socialmente construídas da assistência prénatal

Prevenção

Acolhimento

Aprendizagem

2. Obstáculos que interferem na nãoadesão ao prénatal
Ausência de rede de apoio

Desorganização dos serviços
Saber como é que está o bebê, ter cuidado para não correr nem um tipo de risco para saber como vai correr todo meses, ou algum risco de alguma coisa. Para me prevenir, prevenir o bebê (E1). Pré-natal é importante por causa da saúde do bebê e a minha também! Porque se eu tiver com alguma doença pode passar para o bebê! (E5).

O pré-natal foi muito importante, me ajudou bastante no momento que eu estava precisando, porque tive um problema na gravidez, eu ia pro posto, eles me tratavam muito bem, me acolhia, sei que me deram muita segurança lá (E9). Já é o quarto filho! E eu tive esses problemas de pressão, aí você sabe que tem que cuidar(E4). Meu primeiro filho nasceu prematuro. Com isso, eu vim, tem que evitar novamente outro parto prematuro(E8).

Com o pré-natal descobri várias coisas que eu não sabia. O que a gente deve fazer com os bebês (E10). Eu acho importante! Porque nós sabemos o que pode comer para não sentir enjoo e para tomar os remédios de forma correta $(\mathrm{E} 7)$.

Eu não fui mais fazer [o pré-natal] porque tenho três meninos, daí, você sabe, é difícil ter com quem deixar. O pai deles, meu marido, tá lá no barranco [garimpo]. Aqui não tenho ninguém, nem uma irmã pra ficar olhando eles, não dá pra descer o rio com eles sempre. (E4). Eu ainda tava na escola, não tinha com quem contar, minha mãe não aceitou eu estar grávida de jeito nenhum. Aí eu e o pai dele tivemos que trabalhar e não tive como ir no posto (E7).

A falta de dinheiro para realizar exames particulares que não tem aqui (E8). Eu sou do Cassiporé, lá não tem exame pra nada, pra eu vir pra cidade gasto dinheiro aqui e não resolvo nada. Não tem como tá todo mês aqui [sede urbana], não! (E2). É ruim porque não tem os remédios que a doutora passa, aí a gente fica de um lado pro outro e chega a ter 0 menino e não recebe nada (E1).

Meu marido é pirateiro e eu sou marreteira [comerciante móvel], né? Aí eu trazia umas coisa de Goiânia e de Fortaleza pra vender aqui [Oiapoque]. Às vezes eu tava lá [Macapá], às vezes no mundo[risos], às vezes eu tava aqui [Oiapoque]. Não tive como fazer pré-natal direitinho não, mas aí nasceu prematuro[choro](E8). Ela só fez uma consulta aqui na cidade, com a enfermeira, lá na aldeia só tinha médico e o marido dela não deixava ela ir pra consultas porque ele era homem (E11).Tem também a nossa língua, né? Só os homens e as velha que falam português, aí tem que ir acompanhada (E12)

Fonte: Dados da pesquisa, 2021. 


\subsection{Percepção Socialmente Construídas da Assistência Pré-natal}

A primeira categoria codificada a partir das falas versou sobre a percepção que as entrevistadas possuem sobre 0 pré-natal. As representações foram ancoradas em diversos eixos, os quais se destacaram a prevenção de agravos à saúde materna e infantil, a aprendizagem sobre o maternar e os cuidados com o bebê e o acolhimento recebido durante o período gravídico puerperal.

A prevenção surgiu como uma das principais percepções do atendimento. A prevenção de agravos com o concepto e recém-nascido, durante o parto e a transmissão vertical de doenças fizeram parte das representações. Essa categoria também foi relacionada à precaução de intercorrências, verbalizada principalmente por multíparas com históricos gestacionais pregressos de complicações. A prevenção como motivação à realização do pré-natal foi observada em outros estudos, principalmente no que concerne a preocupação com o crescimento e desenvolvimento de um bebê saudável. Esta apreensão pode estar associada aos elevados índices de morbimortalidade durante o período neonatal precoce da região, que é superior à média nacional (Gama, Fernandes, Parente, \& Secoli, 2018). A representação social acerca da assistência pré-natal centrada à saúde do bebê ancorase na abordagem realizada no início das políticas de saúde materno-infantil, em que a ênfase do cuidado estava circunscrita ao útero gravídico, tornando aspectos psicossociais da saúde materna marginalizados ao cuidado.

Outro ponto elencado foi o comparecimento às consultas motivado pela aquisição de conhecimentos sobre os cuidados a serem realizados com bebê e para extrair dúvidas acerca de uma nutrição adequada e sobre o uso apropriado de medicações e suplementos. O pré-natal é um período em que há oportunidade de preparação à maternidade, em que há a possibilidade de um planejamento de cuidados e oportunidade de aprendizagem. No entanto, é possível observar na literatura que mulheres de baixa renda, assim como mulheres residentes da região Norte e de municípios de pequeno porte recebem menos orientações e ações educativas adequadas durante o pré-natal (Tomasi et al., 2017). Todavia, as representações das participantes deste estudo podem ser apontadas como um reflexo positivo sobre a qualidade da assistência, em que as participantes observaram no pré-natal um espaço à discussão de suas dúvidas.

A resposta às necessidades dos usuários dos serviços de saúde é um dos eixos propostos pelo acolhimento seja por meio da assistência propriamente dita ou através da disponibilização de informações. De acordo com (Gonçalves, Souza, Amaral, Oliveira, \& Ferreira, 2017), o acolhimento é parte estruturante e agente determinante para o sucesso da assistência pré-natal, uma vez que fortalece a relação dos profissionais de saúde com a comunidade e seus diversos contextos de atuação. Ele contribui com as diretrizes operacionais para a materialização dos princípios do SUS, destacando a integralidade, universalidade e equidade em saúde. $O$ acolhimento também esteve ancorado nos discursos das puérperas e percebido como uma das motivações à adesão e continuidade das consultas de pré-natal, em que as puérperas relataram a importância do vínculo para formulação de um sentimento de segurança e autoconfiança. Observa-se, assim, a importância de disponibilizar um canal de diálogo acessível às gestantes e puérperas, respeitando os valores e limitações que envolvem seus contextos socioculturais

\subsection{Obstáculos que Interferem na Não-adesão ao Pré-natal}

A segunda categoria identificada refere-se aos principais obstáculos a que as entrevistadas vivenciaram e desvelaram como representações. Estas representações foram ancoradas na ausência de redes de apoio social. A partir do suporte emocional e psíquico de pessoas próximas à gestante, a mulher sente-se mais segura e acolhida. $\mathrm{O}$ apoio de familiares, amigos, vizinhos e profissionais de saúde são imprescindíveis à adesão das consultas pré-natais, podendo configurar-se como um determinante na adesão e na manutenção da assistência. A literatura verifica que os processos de cuidado que envolvem a família aumentam a preocupação pelo bem-estar de si e do bebê. 
O apoio social é uma ferramenta de suporte psicológico e emocional à maternidade, capaz de gerar condições satisfatórias e facilitadoras de interação entre os novos pais e o bebê, capacitando-os ao exercício de suas novas funções como cuidadores. O apoio é de extrema importância ao enfrentamento de situações estressantes, além da adequação de comportamentos maternos em relação aos filhos Gonçalves et al., 2017.

A peregrinação relacionada à distância das Unidades Básicas de Saúde, a morosidade para marcação de consultas e exames, a dificuldade de acesso à dispensa de medicações, a ausência de recursos financeiros para acessar exames não ofertados no município também foram evidenciadas nas falas com obstáculos à adesão precoce e seguimento das consultas. É importante salientar que o referido município conta com um serviço definido na política de atenção à saúde indígena, Casa de Saúde Indígena (CASAI), no qual oferece apoio às gestantes indígenas que precisam de assistência de saúde na sede. No entanto, estas gestantes, geralmente realizam a assistência pré-natal nas unidades de saúde em suas aldeias, utilizando a CASAI como espaço de apoio apenas quando necessitam de assistência hospitalar. Entretanto, na configuração municipal não há serviços de apoio para acolhimento de gestantes oriundas de outras áreas rurais, distantes da sede do município em que não verifica-se serviços de saúde como as unidades básicas de saúde. Muitas destas gestantes moram em áreas de difícil acesso onde não há serviços de saúde próximos, a exemplo de áreas de garimpos e assentamentos. Neste sentido, estudos apontam que uma situação socioeconômica desfavorável e de difícil acesso demográfico, como as verificadas neste estudo, dificultam o acesso das gestantes às consultas pré-natais (Silva, Mendes, Monteiro, \& Silva, 2019). A distância física e o tempo de viagem são classificados como barreiras relacionadas ao acesso referentes à organização dos serviços. As barreiras geográficas também podem atuar como agentes limitantes à aquisição de informações epidemiológicas dessas populações. Coadunando com estes relatos, verifica-se que a situação de isolamento geográfico ainda afeta a atenção à saúde da mulher na Amazônia. Estudo realizado na Amazônia Peruana demonstrou que a localização da residência se mostrou significativamente associada ao atendimento pré-natal no primeiro trimestre, em que as residências rurais obtiveram menor acesso (Moore, Blouin, Razuri, Casapia, \& Gyorkos, 2017).

Considerando que o SUS orienta a universalidade e integralidade na oferta de serviços, a existência de custos diretos à realização da assistência pré-natal, a exemplo do financiamento por parte das puérperas à realização de exames inexistente no escopo de programas de saúde do município, como também os gastos indiretos, a exemplo de transporte, hospedagens e alimentação, devem ser observados para análise e reorganização da estrutura dos serviços. Estes custos atuam aumentando as desigualdades sociais no acesso à assistência de saúde, em que gestantes mais pobres são mais atingidas (Esposti, Oliveira, Santos Neto, \& Travassos, 2015).

Os aspectos socioculturais também estiveram ancorados nas representações dos obstáculos à adesão ao pré-natal. Entre estes aspectos foram elencadas as condições de trabalho presentes no contexto de fronteira amazônica, principalmente as que exercem um grande fluxo nesta região, a exemplo de garimpeiros. A garimpagem é uma das principais fontes de recursos financeiros desta região; nos quais, na maioria das vezes, 0 acesso aos garimpos permeia cerca de 70 horas de viagem da sede urbana. A necessidade de continuar trabalhando emergiu como obstáculo à adesao. Nesta conjuntura, se destaca que a universalização do acesso deve ser direcionada à redução das barreiras físicas e financeiras. O tempo de espera relacionado ao sistema de marcação de consultas e exames, assim como a distância física e as relações de trabalho surgem como obstáculos à universalidade da assistência pré-natal no relato das entrevistadas. A gestante garimpeira precisa ausentar-se de sua atividade laboral, perdendo sua única fonte de renda. Estas mulheres também, muitas vezes, encontram-se em condições de clandestinidade, trabalhando em garimpos clandestinos situados em áreas de floresta na Guiana Francesa, sem documentos de registo civil, o que as impedem de receber auxílios de transferência de renda do governo brasileiro. 
A região fronteiriça de Oiapoque-Tumucumaque também é numerosa em etnias indígenas e em outros povos da floresta. Evidências recentes em relação às iniquidades sociais presentes nos processos de nascimento remontaram à diversidade étnica cultural do Brasil, em que indígenas apresentaram maior probabilidade de desenvolver near misses (Fernandes, Souza, Leal, Moura, Santos, \& Cecatti, 2017). Destaca-se, ainda, que durante as entrevistas, a mãe de uma puérpera foi a intérprete da entrevista, uma vez que a puérpera, da aldeia Kumenê, da etnia Palikur, não compreendia o idioma português, somente o parikwaki, filiada à grande família linguística Arawak. Desta forma, a diversidade cultural foi também observada como fator limitante à comunicação efetiva, em que as numerosas etnias indígenas presentes na região do Amapá e Norte do Pará inseriram-se neste contexto, além de estrangeiros, e, muitas vezes, o idioma falado tornase uma barreira à comunicação entre a gestante e o profissional e, por conseguinte, um determinante à adesão tardia e/ou descontinuidade das consultas. O dialeto se torna um agente limitante à compreensão real do que a paciente refere sobre sua saúde, por mais que haja intérpretes (Silva et al., 2016). A tradução literal resulta, algumas vezes, em equívocos, em que há palavras que não possuem significante em outro idioma, principalmente mediante o vocabulário técnico referente à área de saúde.

Entre algumas mulheres indígenas foi observado que a realização do pré-natal está condicionada a autorização do pai da criança, que proíbe a realização da assistência quando se trata de um profissional de saúde do sexo masculino. A narrativa elaborada por mulheres requer uma compreensão entre outros aspectos das questões associadas ao gênero. O status de acesso aos serviços de saúde congregam papeis comuns a todas as pessoas, indiferente ao gênero. Entretanto, o percurso e o acesso aos serviços são vivenciados distintamente e os significados atribuídos assumem contornos assinalados por questões diferentes entre si, neste caso, ser mulher.

\section{Conclusões}

As representações sociais inferem que a prevenção, o acolhimento e a aprendizagem são as principais percepções acerca da assistência pré-natal. Os obstáculos à adesão inserem-se nos contextos dos aspectos sócioculturais vivenciados no cotidiano fronteiriço amazônico, a respeito das precárias relações trabalhistas, violência de gênero, variabilidade linguística e culturais; a ausência das redes de apoio; além da desorganização dos serviços, que se encontram distantes, têm morosidade quanto ao sistema de marcação e possui restrições que necessitam de aporte financeiros pelas usuárias.

Ressalta-se a importância de estruturação da rede de assistência a fim de conhecer as necessidades da população assistida, a exemplo da ampliação da cobertura, da capacitação dos profissionais para mediação dos conflitos relacionados ao gênero e para o trabalho em áreas de grande variabilidade cultural, como as regiões amazônicas e de fronteira, para assim poder oferecer adequação dos serviços de saúde. Destaca-se que as representações apontadas podem direcionar pesquisas futuras acerca da adesão ao pré-natal, em que se destaca o aprofundamento das temáticas do acompanhamento de gestantes com redes trabalhistas precárias e da capacitação dos profissionais de saúde para assistência no contexto de diversidades étnicas e culturais.

Como limitação do estudo aponta-se o fato da pesquisa ter sido realizada em único cenário da referida cidade. Desse modo, deve-se considerar que, frente à realidade da Floresta Amazônica, os dados não podem ser generalizados; visto que, muitas mulheres, em especial as indígenas e ribeirinhas, vivenciam o parto domiciliar. Podemos afirmar que as comunidades indígenas presentes na região Oiapoque-Tumucumaque representam etnias que diferem muito entre si. Neste sentido, a limitação ou interdição da liberdade das mulheres não podem ser generalizada como presente em todas as etnias. Salienta-se que outra condição limitante foi o fato de não terem sido captadas as puérperas que apresentaram near misses no último ciclo gravidico-puerperal; pois estas são encaminhadas à atenção terciária de referência estadual, localizada na capital Macapá, ou ainda, direcionadas ao Hospital de Cayenna, na Guiana Francesa. 
As contribuições da metodologia qualitativa nesta abordagem inserem-se no conhecimento das representações sociais que impactam diretamente a adesão à consulta do pré-natal entre povos da floresta. Nota-se que estudos dessa natureza são essências para desvelar os fenômenos vivenciados por sujeitos que vivenciam a atenção pré- natal em regiões consideradas vulneráveis, favorecendo a avaliação e direcionamento de políticas públicas nacionais e internacionais.

\section{Referências}

Ansu-Mensah M., Danquah F.I., Bawontuo V., Ansu-Mensah P., Kuupiel D (2020). Maternal perceptions of the quality of Care in the Free Maternal Care Policy in sub-Sahara Africa: A systematic scoping review. BMC Health Services Research, 20. Doi: 10.1186 / s12913-020 05755-9.

Esposti, C., Oliveira, A., Santos Neto, E., \& Travassos, C. (2015). Representações sociais sobre o acesso e o cuidado pré-natal no Sistema Único de Saúde da Região Metropolitana da Grande Vitória, Espírito Santo. Saúde e Sociedade, 24(3), 765-779. Doi: 10.1590/S010412902015127606

Fernandes, K. G., Souza, R. T., Leal, M. C., Moura, E. C., Santos, L. M., \& Cecatti, J. G. (2017). Ethnic differences in maternal near miss. Archives of gynecology and obstetrics, 296(6), 1063-1070. Doi:10.1007/s00404-017-4530-6

Gama, A., Fernandes, T., Parente, R., \& Secoli, S. (2018). Inquérito de saúde em comunidades ribeirinhas do Amazonas, Brasil. Cad Saude Publica, 34(2). Doi: 10.1590/0102$311 \times 00002817$

Guimarães, W., Parente, R., Guimarães, T., \& Garnelo, L. (2018). Acesso e qualidade da atenção pré-natal na Estratégia Saúde da Família: infraestrutura, cuidado e gestão [Access to prenatal care and quality of care in the Family Health Strategy: infrastructure, care, and management]. Cadernos de saude publica, 34(5), e00110417. Doi: 10.1590/0102$311 \times 00110$

Gonçalves, I., Souza, K., Amaral, M., \& Oliveira, A., Ferreira, W. (2017) Prática do acolhimento na assistência pré-natal: limites, potencialidades e contribuições da enfermagem. Rev Rene. 14(3), 620-9. Disponível em: http://www.periodicos.ufc.br/rene/article/view/3503 .

Moore, N., Blouin, B., Razuri, H., \& Casapia, M., Gyorkos, T.W. (2017). Determinants of first trimester attendance at antenatal care clinics in the Amazon region of Peru: A case-control study. Montazeri A., editor. PLoS One, 16;12(2):e0171136. Doi: 10.1371/journal.pone.0171136

Moscovici S. (2003). Representações sociais: investigações em psicologia social. Petrópolis, RJ: Vozes.

Tomasi, E., Fernandes, P., Fischer, T., Siqueira, F., Silveira D., Thumé E. (2017). Qualidade da atenção pré-natal na rede básica de saúde do Brasil: indicadores e desigualdades sociais. Cad Saude Publica, 33(3). Doi: 10.1590/0102-311x00195815

Silva, D., Nascimento, E., Santos, L., \& Martins, N., Sousa, M., Figueira M. (2016). Dificuldades enfrentadas pelos indígenas durante a permanência em uma Casa de Saúde Indígena na região Amazônica/Brasil. Saúde e Soc, 25(4):920-9. Doi: 10.1590/s0104-12902016160600

Silva, A., Mendes, L., Monteiro, R., Silva, R. (2020). Parto e nascimento na fronteira francobrasileira: percepções de enfermeiros. Revista Cogitare, 25: e67820. Doi: $10.5380 /$ ce.v25i0.67820 